\title{
Überlegungen zu einer theologischen Kommunikationstheorie
}

\author{
von Karl Grüner
}

Kommunikation ist ein Schlagwort unserer Tage, das auch im kirchlichen Bereich zunehmend Verwendung findet. Man betrachtet die Predigt unter kommunikativen Aspekten, spricht von Kommunikation in der Pfarrgemeinde, analysiert kommunikative Prozesse im Pfarrgemeinderat und hat sich daran gewöhnt, nicht mehr $\mathrm{zu}$ "sprechen «, sondern $\mathrm{zu}$ »kommunizieren«. Über diesen undifferenzierten Gebrauch hinaus setzt sich auch die Theologie ernsthaft mit der Theorie kommunikativen Handelns im Bereich des Glaubens und der Kirche auseinander. ${ }^{1}$ Nicht zuletzt sind viele, die das Kommunikationsgeschehen in der Kirche beruflich als Journalisten oder Publizisten oder ehrenamtlich in der gemeindlichen Öffentlichkeitsarbeit mitgestalten, an einer tieferen Sinngebung ihrer Tätigkeit interessiert. Im folgenden soll deshalb versucht werden, den von den Sozialwissenschaften geprägten Kommunikationsbegriff auf seine theologischen Implikationen hin zu untersuchen. Legt man den neuen wissenschaftlichen Ansatz von Helmut Peukert zugrunde, so kann man Theologie sogar als »Theorie des kommunikativen Handelns und der im Handeln erschlossenen und erfahrenen Wirklichkeit Gottes ${ }^{2}{ }^{2}$ verstehen. Das bedeutet, daß in der menschlichen Kommunikation und durch sie auch etwas von Gottes Wirklichkeit aufscheint und es heißt ferner, daß auch von Gott kommunikatives Handeln ausgesagt werden kann. Damit erhält die Kommunikation geradezu theologische Qualität.

Im ersten Teil soll versucht werden, Kommunikation von der "communio « mit Gott, einem Kernbegriff des II. Vatikanischen Konzils, theologisch abzuleiten und so zu einer theologischen Begriffsbestimmung von Kommunikation zu finden. Partner der communio Gottes und damit Ziel seines kommunikativen Handelns ist der Mensch, den Gott nach seinem Bild geschaffen hat, um sich ihm mitzuteilen. Dies soll im zweiten Teil des Beitrages weiter entfaltet werden.

\section{Theologischer Kommunikationsbegriff}

Das Zweite Vatikanische Konzil betont im Missionsdekret, daß Gott »uns in seiner übergroßen Barmherzigkeit und Güte aus freien Stücken geschaffen und überdies gnadenweise gerufen (hat), Gemeinschaft zu haben mit ihm in Leben und Herrlichkeit ${ }^{3}$. Und in der Pastoralkonstitution Gaudium et Spes führt das Konzil aus: "Gott rief und ruft nämlich den Menschen, daß er ihm in der ewigen Gemeinschaft unzerstörbaren göttlichen Lebens mit seinem ganzen Wesen anhange. ${ }^{4}$ Darin drückt sich die Berufung des Menschen zur Communio mit Gott aus. Gott selbst ist ja Gemeinschaft. Seine Seinsweise ist die communio, die sich im liebenden Ineinandersein der drei göttlichen Personen realisiert. Und an dieser communio will Gott dem Menschen Anteil geben. Deshalb hat er ihn »nach seinem Bild « erschaffen und hat sogar seinen Sohn Mensch werden lassen, um so die menschliche Natur in die göttliche communio miteinzubeziehen. In Christus, dem neuen Adam, ist das ganze Menschengeschlecht bereits stellvertretend in diese göttliche communio aufgenommen. Er ist die Verleiblichung und gleichzeitig auch die Garantie der communio zwischen Gott und Mensch. Das Erschaffensein des Menschen nach Gottes Bild befähigt ihn, auch schon in seiner diesseitigen Existenz Gemeinschaft herzustellen, mit anderen Men-

Karl Grüner ist Diplomtheologe und Redakteur der Münchener Katholischen Kirchenzeitung. 
schen in communio zu treten - freilich aufgrund der Geschöpflichkeit und Endlichkeit des Menschen nur in vorläufiger, unvollkommener Form. Die endgültige communio, die die Menschen einmal mit Gott und in ihm vereint, wird durch communicatio antizipiert. Jede Kommunikation, die auf Gemeinschaft mit anderen Menschen zielt, verweist auf die göttliche communio, nimmt sie zeichenhaft vorweg und trägt im Rahmen des eschatologischen Vorbehalts zu ihrer endgültigen Verwirklichung bei. Dadurch leistet die Kommunikation einen "Beitrag zur Erfüllung des göttlichen Heilsplans in der Geschichte", wie es in der Pastoralinstruktion 'Communio et Progressio' heißt. ${ }^{5}$ Papst Paul VI., auf dessen Gedanken zur sozialen Kommunikation die Pastoralinstruktion vor allem aufbaut, sieht das Ziel der menschlichen Kommunikation im Einander-Näherrücken der einzelnen Menschen und der ganzen Menschheit und erblickt darin einen Beitrag zur Verwirklichung des Heils, das in der communio im Reiche Gottes besteht ${ }^{6}$. Menschlichkeit, Brüderlichkeit, Solidarität sind für ihn Ziele jeder Kommunikation. Diese sind aber keine rein humanen, irdischen Werte; er sieht sie vielmehr rückgebunden in die Gottesgeschöpflichkeit und Gotteskindschaft des Menschen: „Christen werden nicht vergessen, daß die Bruderschaft, die sie mit den übrigen Menschen verbindet, ihre Wurzeln im Anteil an der göttlichen Sohnschaft hat. Ursprung und Ziel aller höheren Werte ist der lebendige Gott und zugleich auch ihr Bürge. « ${ }^{7}$

\subsection{Kommunikation und Glaube}

Wo immer Christen miteinander oder mit der »Welt $«$ kommunizieren und wo diese Kommunikation gelingt, bezeugen sie, daß Gott an ihnen handelt, Gott, der Urquell jeder Kommunikation. Dieses Bezeugen hat mit Glauben zu tun. Zwischen Kommunikation und Glaube besteht eine Korrelation, ja "Glaube ist Kommunikation «, wie Klaus Hemmerle formuliert ${ }^{8}$. „Er ist das Gegenteil einer bloßen Theorie über Gott; Er ist die Überzeugung, daß Gott selber an uns handelt, daß er unmittelbar zu uns ist und daß wir unmittelbar sind zu ihm, wir zu ihm reden dürfen, er uns hört, er auf uns eingeht, ja er in seinem menschgewordenen Sohn, in seinem Kreuz an uns leidet. «9

Über diese unmittelbare Beziehung zu Gott, die die vertikale Dimension des Glaubens anzeigt, hinaus, ist Glaube auch angewiesen auf die horizontale Vermittlung, auf die Kommunikation im Wir. Diese ist die ekklesiologische Dimension des Glaubens und der Kommunikation ${ }^{10}$. Diese beiden Aspekte der Glaubenskommunikation sollen noch etwas weiter entfaltet werden.

\subsubsection{Glaube als vertikale Kommunikation mit Gott}

Der Glaube des alttestamentlichen Gottesvolkes gründet auf dem Bundesschluß mit Jahwe. Eräußert sich in der Anerkennung Gottes als Herrn, als der er sich durch seine Machttaten erwiesen hat, im Gehorsam gegenüber seinen Weisungen, in der Treue zum Bund, in der Hoffnung auf das Eintreffen der Verheißungen Gottes und so weiter. Der Glaube Israels ist kein unpersönlicher, kein kognitiver Akt, sondern ein Akt der Beziehung, der Kommunikation mit Jahwe auf personaler Ebene. Und er ist ein kollektiver Akt, weil der Glaube des einzelnen eingebunden ist in den Glauben des Volkes, mit dem Jahwe seinen Bund geschlossen hat. Diese Merkmale des alttestamentlichen Glaubens begegnen uns wieder im Neuen Testament. Auch der christliche Glaube besteht nicht zuerst und wesentlich im Fürwahrhalten der Wahrheiten, die Christus geoffenbart hat - wiewohl dieser Aspekt nicht auszuschließen ist -, sondern in einem grundlegenden Akt der Anerkennung, des Vertrauens, der Liebe. Glaube ist umfassender Ausdruck für das Verhältnis des Menschen zu Gott, das aus der Annahme der frohen Botschaft erwächst. Er ist die Reaktion des Menschen auf den Anruf Gottes, der ihn in Jesus Christus unmittelbar erreicht. Es handelt 
sich um ein kommunikativ-dialogisches Geschehen: Gott ruft und beruft den Menschen. ${ }^{11}$ Dieser hört und empfängt den Anruf Gottes und gibt seine Antwort, jedoch nicht primär in der Form einer rationalen Zustimmung zu den Inhalten der Offenbarung, sondern in einem personalen Ja zu Gott, das mehr bedeutet als verstandesmäßige Übereinstimmung mit den geoffenbarten Wahrheiten.

Dennoch ist das eigene Denken, die geistige Einsicht in die kognitiven Inhalte der Offenbarung nicht überflüssig. Metanoia muß ja ein freier Akt der Umkehr und Hinwendung zu Gott sein. Freiheit aber gründet auf Einsicht. So kommt zwar nicht das Erkennen aus dem eigenen Nachdenken, wohl aber die Zustimmung des Menschen zu dem Erkannten. So beinhaltet der Glaube, den wir als kommunikativen Akt des Anrufens von seiten Gottes und der Antwort auf seiten des Menschen betrachtet haben, zwei Dimensionen: die Dimension des »Du«, der Beziehung zu Gott als Person und die Dimension des »Daß« oder des Inhalts der Offenbarung. ${ }^{12}$

Dabei stoßen wir auf zwei Aspekte, die jeder Kommunikation und damit auch dem Glauben als Kommunikationsgeschehen zugrunde liegen: den Inhalts- und den Beziehungsaspekt. Paul Watzlawik kommt das Verdienst zu, den Unterschied zwischen beiden und ihre wechselseitige Bezogenheit aufeinander auf sozialpsychologischer Ebene herausgearbeitet zu haben: Der Inhalt einer Kommunikation ist die Information, die der Sender dem Empfänger übermitteln will. Die Art der Übermittlung, die Formulierung, begleitende Mimik und Gestik und meist schon der Inhalt selbst sagen aber auch etwas über die Beziehung zwischen Sender und Empfänger aus und definieren diese Beziehung bei jedem Kommunikationsvorgang von Neuem. ${ }^{13}$ Der Inhaltsaspekt tritt dabei hinter den Beziehungsaspekt zurück, nach Watzlawik derart, daß die Beziehung zwischen den Kommunikationspartnern den Inhalt ihrer Kommunikation bestimmt. Er räumt allerdings ein, daß man rein logisch auch das Umgekehrte sagen könnte, nämlich "daß eine Klasse (Menge) von ihren Elementen - und daher die Beziehung vom Inhaltsaspekt - bestimmt wird. «14

Zwischen beiden Aspekten besteht eben eine Wechselwirkung. Es ist nicht zu leugnen, daß der Inhalt einer Kommunikation eine Reaktion des Empfängers auf der Beziehungsebene determiniert. Es ist aber ebenso zutreffend, daß die bereits vorhandene Beziehung (bei einander Unbekannten ist das Sich-nicht-Kennen die Art der bestehenden Beziehung) schon die Wahl des Kommunikationsinhalts beim Sender beeinflußt. Die zeitliche oder logische Priorität zwischen beiden Aspekten ist jedoch letztlich zweitrangig. Entscheidend ist, daß jede Kommunikation, sie mag noch so belanglos oder noch so sachbezogen sein, die Beziehung zwischen den Partnern je von Neuem definiert. Das Ergebnis des Kommunikationsprozesses ist daher stets eine wie auch immer geartete Beziehung. Darin liegt das spezifisch humane der Kommunikation zwischen Menschen, daß sie sich selbst mitteilen, wenn sie etwas mitteilen. Computer können nur auf der Inhaltsebene Informationen austauschen. Sie bringen dabei keine Beziehung hervor, es sei denn, sie wird ihnen vorher eingespeist, wie beispielsweise eine mathematische Funktion, die eine Beziehung zwischen Zahlen herstellt. Menschliche Kommunikation dagegen zielt grundsätzlich auf Beziehung.

Das kann so weit gehen, daß die Inhalte immer unwichtiger werden, je intensiver sich die Kommunikation zwischen den Menschen gestaltet. In der vollendetsten Kommunikation, in der Liebe zwischen zwei Menschen, kann der Inhalt ganz in der Beziehung aufgehen. Die Liebe braucht keine Inhalte mehr, um sich mitzuteilen. Die Beziehung selbst wird zum Inhalt. Darin aber kommt sie der göttlichen communio am nächsten. Auch in Gottes Selbstmitteilung an den Menschen sind Inhalts- und Beziehungsaspekt identisch. »Inhalt « 
der Kommunikation Gottes mit dem Menschen ist seine Beziehung zu ihm, die in der Liebe besteht.

\subsubsection{Glaube als horizontale Kommunikation unter den Menschen}

Glaube besteht jedoch nicht nur in der vertikalen Begegnung des Individuums mit seinem Gott. Die horizontale Begegnung mit dem Mitmenschen muß hinzukommen. Das bedeutet keine Reduzierung des Christseins auf reine Mitmenschlichkeit. Das wäre nur der Fall, wenn sich die Beziehung zum Nächsten herleiten würde aus irgendeinem Humanitätsgefühl, aus Sympathie, Erotik oder einfach aus dem Erfahrungswissen, daß zum Überleben die Beziehung zu anderen Menschen notwendig ist. Bloße Mitmenschlichkeit, auch wenn sie von Christen geübt wird, ist noch kein horizontaler Glaube. Das wird sie erst, wenn sie als integrierender Bestandteil der vertikalen Kommunikation mit Gott gesehen wird. Biblisch betrachtet sind die Menschen durch die Geschichte, die Gott mit ihnen hat, untereinander verbunden. Dadurch, daß Gott sich mit dem Menschen eingelassen hat, vom Schöpfungsakt an, dem die Welt und die ganze Menschheit sich verdanken, über den Bund mit dem Volk Israel bis zu seiner eigenen Menschwerdung in Jesus Christus, ist die Menschheit aufs engste mit ihm verbunden. Das bedeutet auch, daß der Mensch im Mitmenschen dem Wirken Gottes begegnet und daß die Begegnung mit dem Mitmenschen erst von Gott her ihren eigentlichen Sinn erfährt. Aus der vertikalen Gottbegegnung bezieht die horizontale Kommunikation mit den Mitmenschen ihre Sinngebung. Aber ebenso bedarf die vertikale Begegnung mit Gott der notwendigen Ergänzung und Bewährung in der horizontalen Begegnung mit den Menschen. So ist Glaube ein Miteinander und Ineinander, ein Voneinander und Zueinander vertikaler und horizontaler Kommunikation. Letztere darf dabei nicht im bloßen Mitteilen von Inhalten bestehen, sondern im Sicheinander-Mitteilen der Personen.

Als bevorzugter Ort dieser vertikalen und horizontalen Glaubenskommunikation versteht sich von ihrem Wesen her die Kirche. ${ }^{15}$ Das paulinische Bild von der Kirche als Leib macht die Notwendigkeit der wechselseitigen Beziehung der Glieder untereinander und zu ihrem Haupt, zu Christus, überaus sinnenfällig. In der Gemeinschaft der Kirche entsteht und wächst der Glaube des Individuums. Durch die kommunikative Vermittlung in der Familie und in der Gemeinde erlernt zum Beispiel das Kind Glaubensinhalte und erlebt es Glauben als Beziehung. Das Kennenlernen des Glaubens, das nie ein rein kognitives Geschehen ist, ist verbunden mit dem Hineinwachsen in die Glaubensgemeinschaft. Es vollzieht sich in einem kommunikativen Prozeß, an dessen Beginn die kindliche Grunderfahrung des Vertrauens und des Angenommenseins in Liebe durch die Eltern steht. Es setzt sich fort im konkreten Erfahren gelebten Glaubens, der sich im gegenseitigen Geben und Empfangen in der selbstverständlichen familiären, aber auch die Familie überschreitenden Diakonie ausdrückt. Und es erfaßt auch schon erste liturgische Elemente im Einüben des Gebets als einer Form der vertikalen Kommunikation mit Gott. Auch das Erlernen des Daß-Glaubens durch elterliche Unterweisung und Beantwortung erster Sinnfragen des Kindes ist ein kommunikativer Vorgang. Dasselbe gilt für das weitere Hineinwachsen des Kindes und des jungen Menschen in den Glauben durch Kontakte außerhalb der Familie in Kindergarten, Schule und Gemeinde. Das Lernen des Glaubens wird immer auch verbunden sein mit einer Integration in die Gemeinschaft der Glaubenden. Es ist nie ein individuelles Geschehen, sondern immer ein Sozialisations- und damit, weil Sozialisation nicht anders erfolgen kann, auch ein Kommunikationsgeschehen.

Glaube entsteht also in Gemeinschaft, entfaltet sich in ihr und muß auch von ihr getragen werden. So läßt sich als theologisches Ziel der Kommunikation die gegenseitige Selbstmitteilung der Menschen ausmachen, deren Ursprung und Korrektiv die Selbstmitteilung 
Gottes ist und als deren Vollendung die endgültige communio im Reich Gottes anzusehen ist.

\subsection{Tödliche Kommunikationslosigkeit}

Eine theologische Kommunikationstheorie wäre jedoch einseitig, wollte sie nicht auch die Negation der communio = Heil stiftende Kommunikation in die Betrachtung miteinbeziehen. Menschliche Bosheit kann Kommunikation mißbrauchen zur Verwirrung, Verleumdung, Verfeindung, zu übler Nachrede, Haß, Zwietracht (alle diese Begriffe werden im Griechischen mit $\delta \mathrm{i} \alpha \beta \mathrm{o} \lambda \eta$ wiedergegeben. Derjenige, der als Wirkursache dahintersteht, ist der $\delta \mathrm{i} \alpha \beta \mathrm{o} \lambda \mathrm{oc}$, ). Dieser Mißbrauch der Kommunikation führt nicht zu Gemeinschaft, sondern verhindert oder zerstört sie. Und wo zwischen Menschen communio bewußt verhindert oder zerstört wird, kommt auch keine Gemeinschaft mit Gott zustande. Die Folge ist Schuld und in letzter Konsequenz Heillosigkeit, Gottferne, Tod. Sünde und Schuld führen zu ichbezogener Isolation, zu Kommunikations- und Beziehungslosigkeit gegenüber Gott und den Mitmenschen. Diese aber stellen eine tödliche Bedrohung dar. Das macht beispielsweise die Sündenfallerzählung des Jahwisten im zweiten und dritten Genesiskapitel deutlich. Die Strafe für das Vergehen des ersten Menschenpaares gegen Gott besteht in der Vertreibung aus dem Paradies, das der Ort der Gemeinschaft mit Gott war. "In diesem Ausschluß von der Gemeinschaft mit Gott wird dem Menschen seine Todverfallenheit erst recht bewußt, denn hier ist ihm eine wesentliche Grundlage seiner Existenzerfüllung entzogen; hier erfährt er daher zum ersten Male den Einbruch einer lebensfeindlichen Wirklichkeit, die dem Menschen des AT immer Ausdruck des Todes ist. «16

Den Tod selbst empfindet der Mensch ja als absolute Beziehungslosigkeit, als Abbruch der mitmenschlichen Kommunikation. Theologisch betrachtet ist aber gerade das Sterben der Moment im Leben des Menschen, in dem ihn das Kommunikationsangebot Gottes am unmittelbarsten und entscheidendsten trifft. Lehnt er es ab, so wird er auf sich selbst zurückgeworfen in absolute Isolation, in den «zweiten Tod “ (Offb. 21, 8). Nimmt er es an, erweist sich für ihn der Tod als Übergang zu einer neuen, beglückenden Kommunikation, die ihn dann auch wieder auf eine neue Weise mit den Zurückgebliebenen verbindet. „Gott wird, indem er im Tod noch den Menschen beim Namen ruft, sich selbst und damit Gemeinschaft mit ihm und Beziehung zu ihm schenkt, Leben für den Menschen: ewiges, schlechthin sinnvolles und erfüllendes leben «, sagt Zulehner ${ }^{17}$.

\subsection{Kommunikation als Umkehr}

Zulehner wagt deshalb die Gleichung: Beziehung ist Leben, Beziehungslosigkeit ist Tod. ${ }^{18}$ So verstanden ist jede gelingende Kommunikation eine Überwindung der Beziehungslosigkeit und damit eine Umkehr vom Weg des Todes zum Leben, ist je wieder Neuzuwendung und Versöhnung mit den Mitmenschen und damit auch mit Gott, der uns im Mitmenschen begegnet. Wenn Sünde als Störung und als Verlust von Kommunikation gedeutet werden kann, dann ist Umkehr und Versöhnung gleichzeitig auch Wiederaufnahme von Kommunikation, »Befreiung vom Zwang ich-bezogener Isolierung. « ${ }^{19}$

Wenn man alle diese Aspekte zusammenfaßt, dann läßt sich Kommunikation aus theologischer Sicht etwa wie folgt kurz definieren: Kommunikation ist die unter den Bedingungen des erbsündlich verfaßten Menschen noch unvollkommene Aneignung der von Gott gegebenen communio, die als endgültige, eschatologische Existenzweise des Menschen im Aufeinanderzu zwischenmenschlicher Beziehungen mit Hilfe medial vermittelter Inhalte im Glauben antizipiert wird. 


\section{Gottes Kommunikation mit den Menschen}

In der Pastoralinstruktion 'Communio et Progressio' steht: „Nach christlicher Glaubensauffassung ist die Verbundenheit und die Gemeinschaft der Menschen - das oberste Ziel jeder Kommunikation - ursprünglich verwurzelt und gleichsam vorgebildet im höchsten Geheimnis der ewigen Gemeinschaft in Gott zwischen dem Vater, dem Sohn und dem Heiligen Geist, die ein einziges göttliches Leben haben. « $^{20}$

Die bisherigen Ausführungen suchten klarzumachen, daß jede menschliche Kommunikation ihr Urbild und ihren Ermöglichungsgrund in der communio Gottes hat. Dabei wurde die besondere Natur der göttlichen communio in ihrer trinitarischen Ausfaltung, auf die 'Communio et Progressio' hinweist, unberücksichtigt gelassen. Es soll auch im folgenden nicht versucht werden, hier eine »innertrinitarische Kommunikationslehre « zu entwerfen. Gott ist ja unbegreifbar. Auch wenn wir glauben, etwas Adäquatesüber Gottes Wesen ausgesagt zu haben, wissen wir, daß er trotzdem der ganz Andere ist, daß er menschliche Vorstellungskraft immer übersteigt, daß er nicht erfaßbar ist und schon gar nicht in ein von Menschen entworfenes Gedankensystem hineingepreßt werden kann.

So bewundernswert auch die großartige geistige Leistung der scholastischen Theologie ist, nach deren Ergebnis - mit aristotelisch-philosophischen Begriffen ausgedrückt - in Gott drei Hypostasen anzunehmen sind, die jeweils numerisch dieselbe göttliche Wesenheit besitzen und deren Verhältnis zueinander durch innergöttliche Hervorgänge und durch Relationen bestimmt ist,müssen wir trotzdem davon ausgehen, daß Gott so nicht wirklich ist, da sein Wesen über alles menschliche Erkennen hinausgeht. Jeder Versuch, ontologische Aussagen über das trinitarische Wesen Gottes zu machen, ist Stückwerk. Auch wenn die klassische Trinitätslehre der Kirche nichts Falsches über Gott aussagt, so bleibt sie doch hinter der Wirklichkeit Gottes zurück, weil menschlicher Geist, der geschöpflich ist und nicht in die Sphäre des Unendlichen vorzudringen vermag, Gott eben nur menschlich ausdrücken kann.

Dasselbe gilt für den Versuch, das innertrinitarische Geschehen mit Kommunikation erklären zu wollen, wie es das Zitat aus 'Communio et Progressio', das die Gemeinschaft der Menschen - das Ziel jeder Kommunikation - aus der ewigen Gemeinschaft der Trinität herleitet, nahelegen möchte: Die immanente Trinität Gottes ist weder mit philosophischen noch mit modernen soziologisch-kommunikationswissenschaftlichen Begriffen adäquat wiederzugeben. Damit soll nicht von der Hand gewiesen werden, daß Kommunikation und Gemeinschaft Begriffe sind, die unsere Vorstellung vom dreieinigen Gott bereichern und vielleicht auch als Ansatz für eine Neuinterpretation der klassischen kirchlichen Lehre von der immanenten Trinität dienen könnten.

Wir haben vor allem die Schrift zu befragen, wenn wir einen Zugang zu dem Gott finden wollen, der sich in drei verschiedenen Weisen in der Geschichte geoffenbart hat. Die Bibel erschließt uns nicht in ontologischen Aussagen die innerste Natur, das statische, in sich ruhende Wesen des dreieinigen Gottes, sondern sein dynamisches Wirken in der Geschichte, sein Verhältnis zum Menschen und des Menschen Verhältnis zu ihm. ${ }^{21}$ Dabei sind naturgemäß auch Hinweise auf das immanente Wesen Gottes enthalten, das aber dem menschlichen Begreifen nicht voll zugänglich ist. Die dreifache Selbsterschließung Gottes, wie sie uns in der Heiligen Schrift vorliegt, geschieht jeweils in einem kommunikativen Vorgang. Gott stellt Beziehung her zu Menschen, spricht mit ihnen und erweist sich dadurch für sie als kommunizierender Gott. Das aber nicht nur durch den Vorgang der Selbstoffenbarung, sondern auch durch deren Inhalt. Indem Gott sich dem Menschen in 
dreifacher Gestalt offenbart, gibt er sich auch als Gott zu erkennen, zu dessen Wesen die Kommunikation, das heißt, das Miteinander der drei Personen in ihm, gehört.

Beide Aspekte der Kommunikation Gottes, der Vorgang und der Inhalt seiner Selbstmitteilung an uns, werden im folgenden in knapper Form am Beispiel der Heiligen Schrift dargestellt.

Die Selbsterschließung Gottes an den Menschen erfolgt stufenweise. Im Alten Testament offenbart er sich als Schöpfer aller Dinge, als Herr, Vater und Bundespartner des Volkes Israel. Im Neuen Testament begegnet er uns in Jesus als Sohn, der Mensch geworden ist und nach Ostern als Heiliger Geist, der in der Kirche fortlebt. Klaus Kienzler sagt deshalb zu Recht: "Die Heilsgeschichte ist als Wille Gottes zur Kommunikation zu verstehen. "22

\subsection{Der Gott des Alten Testaments}

Am Beginn der Schriften des Alten Testaments begegnet uns Gott als Sprechender: »Da sprach Gott 'es werde Licht!' und es ward Licht. «Sein Wort ist wirkmächtig. Indem er es spricht, geschieht das Gesprochene. Der Schöpfungsakt fällt mit dem Sprechakt zusammen. Mangels eines Partners ist jedoch Gottes Sprechen ein Dialogisieren mit sich selbst: "Laßt uns den Menschen machen", sagt er zu sich (oder wie man aus christlicher Sicht vielleicht spekulieren könnte, zu den anderen Personen der Dreifaltigkeit?). Im Menschen aber erschafft sich Gott am sechsten Tag ein Gegenüber, sein eigenes Bild und Gleichnis, mit dem er in Dialog treten kann. Auch als der Mensch im Sündenfall erstmals dem Gespräch mit Gott ausweicht ("und der Mensch und sein Weib verbargen sich vor Jahwe...«) bleibt Gott mit ihm im Gespräch. Sogar die Vertreibung aus dem Paradies bedeutet keinen Abbruch des Dialogs Gottes mit dem Menschen. Er spricht zu Kain und Abel, zu Noah, Abraham und seinen Söhnen, zu Mose und den Propheten.

Das ganze alte Testament erzählt vom Handeln Gottes in der Geschichte des Volkes Israel, das ein kommunikatives Handeln ist, ein Sich-Einlassen Gottes mit dem Menschen. Höchster Ausdruck der Kommunikation Gottes mit den Menschen des Alten Testaments ist der Bund, den Gott mit Israel schließt und den Alfons Deissler eine »dialogische Erwählungsgemeinschaft « nennt. ${ }^{23}$ Gott erwählt sich sein Volk und übernimmt die Verpflichtung, Israel als sein Eigentum zu beschützen, unter der Bedingung, daß auch das Volk seine eingegangenen Verpflichtungen erfüllt. Diese sind: Der Dienst Jahwes, die Treue zu ihm. Die Befolgung seiner Gebote und Weisungen: Vorschriften des Gottesdienstes, tägliche Opfer, Feier der Feste und heiligen Zeiten, Achtung vor den Eltern, Nächstenliebe, Ehrlichkeit, Achtung vor dem Leben und Eigentum des Mitmenschen und so weiter. Dafür verspricht Gott auch irdische Vorteile: den Einzug ins Gelobte Land, Vermehrung des Volkes, Frieden, Reichtum, Gesundheit, langes Leben, viele Nachkommen und so weiter. Israel ist dadurch an Gott gleichsam vertraglich gebunden, wobei es aber keine frei Wahl hat, diesen Bund abzulehnen. Aus freiem Entschluß hat Gott dieses Volk auserwählt. Er ist alleiniger Urheber des Bundes. Israel kann nur zustimmen und sich verpflichten, die Bundesgesetze zu halten. Gott offenbart sich seinem Volk demnach als Fordernder und als Schenkender. Beides, Fordern und Schenken, sind personale und kommunikative Akte, wie sie beispielsweise auch für das Verhältnis Vater-Kind charakteristisch sind. Der Vergleich: Gott als Vater und Israel als Kind begegnet uns deshalb mehrmals im Alten Testament, um die Beziehung Jahwes zu seinem Volk anschaulich zu machen, so beispielsweise bei Hos 11,1; Jes 64,7 oder Mal 2,10.

Gleiches gilt für das Bild von der Liebe der Mutter zu ihrem Kind in Jes 49,15 und 66,13. Sogar die eheliche Liebe dient als Metapher für die enge personal-kommunikative Beziehung Gottes zu seinem Volk, so bei Hos 2,21 f.; Jer 2,2; Ez 16,8 und Jes 54,5. Der zutref- 
fendste Name für das Verhältnis Gottes zu Israel wäre demnach: Liebe. Gott liebt sein Volk und ist ihm persönlich nahe.

Das kommt auch in dem Namen zum Ausdruck, den Mose im brennenden Dornbusch offenbart: Jahwe, das heißt »Ich bin da (für euch)«. Nach Ratzinger verweist diese Namensbildung auf den persönlichen Gott, "das heißt den Gott, der auf den Menschen hingewandt, selbst personhaft und personbezogen ist. Es ist der Gott, der als der persönlich Seiende mit dem Menschen als Menschen zu tun hat. «24

Das Personale in Gott drückt auch der zweite Gottesname der Bibel aus, der Name »El« (oder im Plural „Elohim «). So wird der "Gott der Väter « genannt. Sein wesentliches Kennzeichen gegenüber den anderen Göttern im Umkreis Israels ist, daß er ein "Personengott" ist und nicht eine Gottheit, die an einen besonderen Ort gebunden ist. Der Gott der Väter wird nicht auf der räumlichen Ebene gesehen, sondern auf der Ebene von Ich und Du.

Auch die Abgrenzung zu üblichen Gottesnamen, wie sie Israels Nachbarn kannten, zum Beispiel Baal (Herr) oder Moloch (König) besagt nach Ratzinger, daß El oder Jahwe nicht in aristokratischer Ferne wie ein Despot regiert, sondern daß er dem Menschen nahe ist.

So offenbart sich der Gott des Alten Testaments als Gott, der sich den Menschen zuwendet, für sie da ist, mit ihnen spricht, ja sogar einen Bund mit ihnen schließt. Das abschließende, endgültige Wort Gottes an die Menschen aber sprach Gott durch seinen Sohn. Darauf weist der Verfasser des Hebräerbriefes hin. "Zu verschiedenen Zeiten und auf mannigfache Weise hat Gott von alters her durch die Propheten zu den Vätern gesprochen; am Ende dieser Tage sprach er zu uns durch seinen Sohn.« (Heb. 1,1).

\subsection{Inkarnation des Logos}

"Der einzig erzeugte Sohn, der an der Brust des Vaters ruht, er hat Kunde gebracht “, schreibt Johannes in seinem Evangelienprolog, in dem er Jesus den »Logos «, das »Wort» nennt, das Fleisch geworden ist, nachdem es schon »im Anfang «, das heißt vor Erschaffung der Welt, also immer, bei Gott war, ja selber Gott war (Jo 1,1 f.). Die Kunde, die frohe Botschaft, die er gebracht hat, ist die Liebe des Vaters, die sich in der Fleischwerdung seines Sohnes erweist. „Diese Kunde, die dieser Sohn bringt, ist ja nichts anderes als sein eigenes Sein vom Vater, sein Wort-Sein, das er 'vor aller Schöpfung' (vergleiche Jo 1,1 -5,10; 17,24; Eph 1,4; 1 Petr 1,20) und schon 'am Anfang' (Jo 1,1) war. Als das Wort Gottes ist er das Ur-Wort schlechthin, und so auch die Ur-Kunde Gottes des Vaters an uns Menschen. ${ }^{25}$ Die Inkarnation ist gerade die Botschaft selbst: Gott hat sich in Jesus total mit dem Menschen eingelassen, um ihn aus der Isolation der Sünde zu befreien und in seine göttliche communio miteinzubeziehen. Durch seine Menschwerdung allein ist die Liebe Gottes zum Menschen schon offen-kundig geworden. Jesus ist die Kunde vom Vater, er ist sein Wort, seine Selbstoffenbarung an uns, er ist »der Logos«. Indem also Johannes für Jesus den Logos-Begriff benützt, drückt er für unser heutiges Verständnis aus, daß der einziggezeugte Sohn, der präexistent beim Vater war, die Mitteilung Gottes an uns Menschen ist, gleichzeitig aber auch der Mitteilende, der uns die endgültige Kunde vom Vater und seinem Reich gebracht hat.

\subsubsection{Jesus als »Meister der Kommunikation «}

Jesus ist vom Vater, von dem jegliche Kommunikation ihren Ausgang nimmt, gesandt, um ihn, das heißt seine Liebe, den Menschen mitzuteilen. Der Sohn, der ganz Beziehung zum Vater ist, kann nach dem Johannesevangelium " von sich aus nichts tun « (Jo 5,19,30), das heißt, er ist als Sohn ganz bezogen aufden Vater, hat sein Sein aus der Beziehung vom Vater her. Johannes läßt deshalb Jesus sagen: „Wenn jemand den Sohn nicht ehrt, ehrt er auch 
den Vater nicht, der ihn gesandt hat .. Wahrlich, wahrlich, ich sage euch: wer mein Wort hört und dem glaubt, der mich gesandt hat, der hat ewiges Leben ... « (Jo 5,24). Während jeder andere menschliche Kommunikator ein Geschehen oder die Aussage eines anderen nur durch seine subjektive Brille gefiltert wiedergeben kann, ist Jesus der vollkommene Kommunikator, da er mit dem Vater eins ist (vergleiche Jo 10,30). Deshalb hört der, der ihn hört, auch den Vater und der ihn aufnimmt, nimmt den Vater auf, der ihn gesandt hat (vergleiche Mt 10,40). Jesus geht ganz darin auf, das Wort des Vaters zu sein. Er schiebt nichts Subjektives, vom Vater Verschiedenes und seine Aussage Verfälschendes dazwischen. Was ein menschlicher Kommunikator in geschriebenem oder gesprochenem Wort, in Bild und Ton wiedergibt, wird immer mit dem Vorbehalt der Ungenauigkeit und NichtAuthentizität aufzunehmen sein. Es erfolgt »ohne Gewähr«. Die Verkündigung Jesu aber ist authentisch und deshalb verbindlich. Das ist der Grund, weshalb die Pastoralinstruktion 'Communio et Progressio' Jesus als »Meister der Kommunikation « bezeichnet: Weil er der perfekte Kommunikator ist: »Er verkündet die göttliche Botschaft verbindlich, mit Macht und ohne Kompromiß. «26

Aber auch in der Beziehung zu seinen Rezipienten erweist sich Jesus als hervorragender Kommunikator. "Er hat die Natur derer angenommen, denen er mit dem totalen Wort seines Lebens und in ihrer eigenen Sprache die Frohbotschaft vermittelt. Er kommt aus der Mitte des Volkes. Er steht, wenn er spricht, auf ihrer Seite. ${ }^{27}$ Er bedient sich des narrativen Stils seiner Zeit und seines Volkes, spricht einfach und verständlich, paßt sich dem Vorwissen seiner Zuhörer an: er holt sie da ab, wo sie stehen. Mit den Intellektuellen seiner Zeit, den Pharisäern, Schriftgelehrten, Hohenpriestern und Ältesten verkehrt er auf ihrer Ebene. Da sie die Schrift kennen, diskutiert er mit ihnen über schwierige theologische Fragen, etwa mit den Sadduzäern über die Auferstehung (Mt 22,23-32 par) oder mit den Pharisäern darüber, wie der Messias Davids Sohn sein kann, wo er ihn doch seinen Herrn nennt (Mt 22,41-46 par) und so weiter.

Jesus paßt sich also dem intellektuellen Niveau seiner Zuhörer an. Das trifft auch zu, wenn er mit dem Volk spricht. Dann wählt er einfache Bilder und Gleichnisse aus dessen Erfahrungsbereich. Er spricht vom verlorenen Schaf, vom Licht, das man auf den Leuchter und nicht unter den Scheffel stellt, vom Sämann, vom Senfkorn im Acker, vom gleichen Lohn für ungleiche Arbeit im Weinberg und so weiter.

Jesus nimmt seine Zuhörer ernst, weil er sie liebt und stellt sich deshalb in seiner Rede ganz auf sie ein. Darin ist er ein nMeister der Kommunikation « und ein Vorbild auch für Kommunikatoren unserer Tage in Publizistik, Unterricht, Verkündigung und so weiter.

\subsubsection{Er selbst ist die Botschaft}

Jesus, der "Logos «, das Wort vom Vater her, ist das, was er verkündet. "Er ist der originäre Inaugurator des wirkenden Wortes schlechthin, und was er verkündet, das ist Gottes 'eigentliches Wort', nämlich er selbst; das ist die Selbstmitteilung endgültiger Gemeinschaft, der communio Gottes mit dem Menschen in ihm und durch ihn. In ihm als dem 'Wort Gottes' fallen Wort, Werk und Person in eins zusammen. «28 Deshalb kann er sagen: »Ich bin der Weg, die Wahrheit und das Leben. «(Jo 14,6). Wer den Weg zum Vater gehen will, muß sich total auf diesen Jesus einlassen, sein Leben an Jesu Leben orientieren, ihm nachfolgen. Es genügt nicht, die Botschaft zu hören und sich intellektuell mit ihr auseinanderzusetzen. Person, Botschaft und Praxis bilden eine unverbrüchliche Einheit im Jesusgeschehen. Die Botschaft vom Gottesreich ist in der Person Jesu Ereignis, personifizierte Gegenwart geworden und verlangt deshalb auch eine personale Antwort: Glaube ohne Rückversicherung, Hoffnung wider alle Verzweiflung, liebende Hingabe in der Totalität 
der Nachfolge. Nur indem wir solchermaßen auf das Hören der Botschaft Jesu, die er selber ist, mit personalem Glauben antworten, werden wir in die Kommunikation mit Gott, die allein Leben gewährt, miteinbezogen. Denn: "Wer dem Sohn nicht glaubt, wird das Leben nicht sehen. « (Jo 3,36).

Jesus ist also Gottes Kommunikationsangebot an uns, der Verkündiger und gleichzeitig der Inhalt der Selbstmitteilung Gottes an die Menschen.

\subsubsection{Jesus ist der "Hörende»}

Bei Johannes sagt Jesus zu den Juden: »Viel hätte ich über euch zu reden und euch zu richten; aber der mich gesandt hat, ist wahr und ich rede von ihm zur Welt, was ich von ihm gehört habe. «(Jo 8,26). Jesus ist der erste und vollkommenste Hörer der Botschaft vom Vater, da er selbst diese Botschaft ist. Aber auch während seiner irdisch-menschlichen, historischen Existenz steht er mit dem Vater in Kommunikation: »Der mich gesandt hat, ist bei mir; er läßt mich nie allein, weil ich allezeit tue, was ihm wohlgefällt. « (Jo 8,29). Jesus hört auf den Vater und ist ihm ge-hor-sam. Gehorsam ist die Folge des Hörens. So zum Beispiel am Ölberg, wo er den Vater bittet, den Kelch an ihm vorübergehen zu lassen, gleichzeitig aber seinen Willen dem des Vaters unterordnet und seinen Tod als vom Vater gewolltes Sühnopfer für die Sünden der Welt gehorchend annimmt. Jesus hört aber nicht nur auf den Willen des Vaters, sondern auch auf die Anliegen, Bitten und Nöte seiner Mitmenschen, für die er stets ein offenes Ohr hat. Dieses Hören galt für den irdischen Jesus ebenso wie es für den erhöhten Christus gilt. Mehr noch als zu seinen Lebzeiten, als er nur zu den verlorenen Schafen Israels gesandt war, ist er der erhöhte Herr der auf die Bitten seiner Brüder Hörende. Johannes teilt dies in seinem ersten Brief mit: »Es ist die Zuversicht, die wir vor ihm haben: wenn wir um etwas im Einklang mit seinem Willen bitten, erhört er uns. Und wissen wir, daß er uns in allem, um was wir bitten, erhört, so wissen wir damit auch, daß wir das von ihm Erbetene erhalten. « (1 Jo 5,14 f.). Diese Zuversicht ist es auch, die die Kirche in ihrer Liturgie immer wieder vertrauensvoll rufen läßt: »Christus, höre uns, Christus erhöre uns!«

So erweist sich Jesus, der Logos, als "Meister der Kommunikation «. Er ist der perfekte Kommunikator, der die Botschaft, die er als Hörender vom Vater empfangen hat, unverfälscht und unverkürzt an uns Menschen weitergeben kann, da er als das »Wort Gottes« in einem unerforschlichen Geheimnis diese Botschaft selber ist. In ihm tauchen ferner göttliche und menschliche Natur ineinander und bewirken die Hereinnahme der menschlichen Natur in die göttliche communio. In Christus ist die Kommunikation Gott-Mensch vollkommen verwirklicht. Er ist deshalb der »exemplarische Mensch«, in dem die Berufung und die absolute Zukunft des Menschen vorgebildet und vorweggenommen sind. Er ist also nicht eine absolute Ausnahme, der ganz andere, sondern er ist »Adam «, die vollendete Form des Wesens Mensch, der als Erhöhter »alle an sich ziehen« wird (vergleiche Jo 12,32). ${ }^{29}$

Anuns aber liegt es, da $\beta$ wir uns in freier Entscheidung auf das Kommunikationsangebot Gottes, das uns in Christus als dem Wort Gottes begegnet, einlassen, indem wir ihm nachfolgen, so zu werden versuchen wie er, der »exemplarische Mensch «, um einmal durch ihn (nicht aus eigener Kraft) zur vollkommenen communio in Gott zu gelangen. "So werden wie er « heißt aber, sich in Liebe mit dem Menschen einlassen, für den anderen dasein. Es heißt also Kommunikation.

Die vertikale Kommunikation mit Gott und die horizontale mit dem Menschen fallen aber für den Glaubenden bereits zusammen in der Feier der Eucharistie. Auf ihre Bedeutung für die communio zwischen Gott und Mensch und der Menschen untereinander weist die 
Pastoralinstruktion 'Communio et Progressio' hin: "Durch die Einsetzung der Eucharistie schenkte uns Christus die höchste Form der Einigung, die den Menschen auf Erden möglich ist, und zwar die Einigung zwischen Gott und Mensch und darum auch die innigste und vollendetste Gemeinschaft zwischen den Menschen selbst.« (Nr. 11).

Gott hat uns aber noch ein weiteres Prinzip der Verbundenheit und Einheit gesandt, den Heiligen Geist. Er, die dritte göttliche Person, bewirkt letztlich unsere Einigung mit Gott, daß auch wir zu Kindern Gottes werden und zu ihm »Abba, Vater « sagen können (vergleiche Röm 8,14-16). ${ }^{30}$

\subsection{Heiliger Geist als communio}

Der dritten göttlichen Person kommt in besonderem Maße eine kommunikative Funktion zu. Nach dem Römerbrief teilt sich Gott den Menschen im Heiligen Geist als Liebe mit (vergleiche Röm 5,5), um auch bei ihnen Liebe zu erzeugen. So kann Paulus im Galaterbrief unter den Früchten des Geistes als erste die Liebe nennen (Gal 5,22). Eng zusammen mit der Bezeichnung »Liebe« hängt auch die Benennung »Gabe«, die uns beispielsweise in der Apostelgeschichte begegnet: "Ihr werdet die Gabe des Heiligen Geistes empfangen.* (Apg 2,38). Der Heilige Geist ist die Gabe Gottes, er ist nach Augustinus Gott als der sich Verschenkende, Gott als Gabe. ${ }^{31}$ Und er ist Gott der Liebe. So ist er demnach Gott als Liebe Gebender, denn die Gabe, die er gibt, ist Liebe. Es ist eine doppelte, aber untrennbar zusammengehörende Liebe: die Liebe des himmlischen Vaters zu seinen Söhnen und die Liebe der Kinder Gottes untereinander. Im Heiligen Geist werden wir ermächtigt, ebenso wie Jesus zu Gott »Vater « zu sagen. Das bestätigt Gal 4,6: »Weil ihr nun Söhne seid, sandte Gott in eure Herzen den Geist seines Sohnes, der da ruft: Abba Vater! « So ist der Heilige Geist das Band, das communio herstellt zwischen dem Vater und den vom Sohn erlösten Menschen, die er dadurch ebenfalls zu Söhnen (beziehungsweise Töchtern) macht. Heribert Mühlen ${ }^{32}$ nennt den Heiligen Geist innertrinitarisch die göttliche Wir-heit und versteht diese als Selbstüberschreitung vom Ich zum Du. Entsprechend müßte sich Geisterfahrung in den erlösten Kindern Gottes ebenfalls als Selbstüberschreitung äußern: Durch Christus auf den Vater und die Mitmenschen hin. Das heißt mit anderen Worten: Geistmitteilung zielt auf Gemeinschaft, auf Kommunikation zwischen Gott und Mensch und der Menschen untereinander. "Schließlich hat Christus uns seinen lebensspendenden Geist mitgeteilt, der das Prinzip der Verbundenheit und Einheit ist. « ${ }^{\mathbf{3 3}}$ Das pfingstliche Sprachwunder ist ein Zeichen dafür, wie der Heilige Geist Kommunikation bewirkt: er baut Barrieren ab, die die Kommunikation erschweren oder verhindern. Er macht, daß alle sich verstehen und dadurch eine Grundlage für Gemeinschaft, für communio, legen können. Das Pfingstereignis zählt deshalb auch mit Recht zu den gemeindestiftenden Faktoren, denen Kirche ihre Entstehung verdankt.

Der Heilige Geist baut Gemeinde auf. Er ist es, der uns in der Taufe in den Leib Christi eingliedert und uns dadurch sowohl in die Gemeinschaft mit Gott als auch in die Gemeinschaft der Glaubenden aufnimmt: "Sind wir doch alle in einem Geiste zu einem Leib getauft, ob Juden oder Griechen, Sklaven oder Freie, und alle sind wir mit einem Geist getränkt.«(1 Kor 12,13).

Zur Gemeinschaft stiftenden, kommt die Gemeinschaft bewahrende Funktion des Heiligen Geistes. Er ist es, der in der Kirche stets gegenwärtig ist, vom Vater und vom Sohn gesandt, um uns in alle Wahrheit einzuführen, uns alles, was vom Vater kommt, kundzutun (Jo 16,13-15) und uns an alles zu erinnern, was Jesus gesagt hat (Jo 14,26). Er wird für immer bei uns bleiben und in uns sein (Jo 14,15-17). So ermöglicht Gott in seinem Geist das Fortbestehen der Gemeinschaft der an ihn Glaubenden. Nachdem der Sohn heimgegan- 
gen ist zum Vater, ist er durch den Geist weiter gegenwärtig in der Kirche als Gabe und als Band der Liebe, das die Gemeinschaft zusammenhält und mit ihm, dem erhöhten Herrn, verbindet. Er ist die Kraft der communio, die die Selbstüberschreitung vom "Ich" zum "Du« im jeweils einzelnen ermöglicht und ihn zum "Wir «, zur Gemeinschaft befähigt. Diese Kraft ist es, die Paulus seinen Brüdern in der Gemeinde von Korinth wünscht, wenn er am Ende seines Briefes schreibt: »Die Gnade des Herrn Jesus Christus, die Liebe Gottes und die Gemeinschaft des Heiligen Geistes sei mit euch allen!« (2 Kor 13,13).

Ein Gedanke sei noch angefügt: Der Heilige Geist ist nicht in der Verfügungsgewalt des Menschen. Er weht, wo er will. Er läßt sich nicht planen, nicht zwingen, nicht garantieren. Das gilt natürlich auch für Kommunikation, Gemeinschaft und Einheit der Menschen, die in ihm ihre theologische Wurzel, ihren Ermöglichungsgrund haben. Das Gelingen von Kommunikation und Gemeinschaft ist nicht davon abhängig, wie perfekt alle Regeln der Kommunikation beachtet werden. Es ist nicht eine Frage der optimalen Methode. Eine unbeholfene, allen homiletischen und rhetorischen Regeln widersprechende Predigt kann mitunter mehr an innerer Wirkung beim Zuhörer hervorrufen als eine noch so brilliante, ausgefeilte, methodisch perfekte Kanzelrede. Ähnliches gilt für therapeutische oder seelsorgerliche Beratungsgespräche, für pädagogische Bemühungen in der Schule, für jede Form publizistischer Tätigkeit und öffentlicher Rede. Der »Erfolg « aller derartigen kommunikativen Bemühungen kann nie garantiert werden. Die Erfahrung des Versagens trotz bester subjektiver Disposition des Kommunikators stellt einen möglicherweise vorhandenen Methodendogmatismus radikal in Frage.

Damit ist nichts gegen Methoden und optimale Bemühungen um Kommunikation auf der Grundlage sozialwissenschaftlicher Erkenntnisse gesagt. Sie sind notwendig und erhöhen die Wahrscheinlichkeit des möglichen Gelingens, getreu nach dem theologischen Axiom, daß die Gnade auf der Natur aufbaut. Eine Garantie für Erfolg bieten sie jedoch nicht. Angesichts der Möglichkeit und häufig erfahrenen Wirklichkeit des Scheiterns kommunikativer Bemühungen sollte jedoch die Hoffnung, ja die Gewißheit bleiben, daß wirkliches Verstehen unter den Menschen aus der Kraft des Geistes gelingen kann. Deshalb sollten wir trotz aller frustrierenden Erfahrungen gescheiterter Kommunikation die Versuche zur Verständigung in der Hoffnung und im Vertrauen auf Gottes Heiligen Geist nicht aufgeben, sondern sie im Gegenteil mit allen zur Verfügung stehenden Mitteln und Methoden wagen, uns aber dabei bewußt sein, daß wir »unnütze Knechte« sind, die »nur tun, was sie schuldig sind « ( $\operatorname{Lk~17,10).}$

\section{Anmerkungen}

1 Einige Untersuchungen als Beispiele: W. Bartholomäus, Evangelium als Information. Elemente einer theologischen Kommunikationstheorie am Beispiel der Osterbotschaft. Zürich, Einsiedeln, Köln 1972; H. Balz, Theologische Modelle der Kommunikation. Bastian-Kraemer-Nida, Gütersloh 1978; H. Albrecht, Kirche im Fernsehen, Hamburg 1974; H.J. Dörger, Religion in Spiegel, Zeit und Stern, Hamburg 1973; G.E. Vogt, Kirche und Fernsehanstalten. Entwicklungen, Konzepte, Programmrealisierungen. Osnabrück 1978; G. Schmied, Pfarrgemeinderat und Kommunikation. Zur Soziologie einer neuen Institution. München 1974; B. Klaus u.a. (Hrsg.), Kommunikation in der Kirche. Predigt-Religionsunterricht-Seelsorge-Publizistik. Gütersloh 1979; H.D. Bastian, Kommunikation. Wie christlicher Glaube funktioniert. Stuttgart, Berlin 1972; E.A. Nida, Message and Mission. The Communication of the Christian Faith. New York 1960. Christof Schorsch, Am Anfang war das Wort - und am Ende ... Umrisse einer Kommunikationstheorie der mystischen Erfahrung. Communicatio Socialis 4/1985, 321-331.

2 Helmut Peukert, Wissenschaftstheorie-Handlungstheorie-Fundamentale Theologie. Analysen zu Ansatz und Status theologischer Theoriebildung. Düsseldorf 1976. 
3 Vaticanum II, Missionsdekret 2 b.

4 Vaticanum II, Gaudium et Spes 18 b.

5 Communio et Progressio (CP) 8. - Eine ausführliche Darstellung der Communio-Theologie von CP und ihre Grundlage in den Texten des II. Vatikanums gibt Klaus Kienzler in 'Kommunikative Theologie' nach dem Vatikanum II, Communicatio Socialis 4/1984, 277-288. Kienzler kommt zu dem Ergebnis, daß „Kommunikation « für die Theologie kein äußerliches Beiwort ist, sondern in die Mitte christlicher Grunddaten und Grundaussagen hineinführt: "Die christliche Botschaft ist in ihren zentralen Glaubensvollzügen und -inhalten selbst kommunikativ verfaßt. « A.a.O. 278.

6 Vgl. G. Deussen, Ethik der Massenkommunikation bei Papst Paul VI., München, Paderborn, Wien 1973, 212-218.

7 Paul VI., Botschaft zum 2. Welttag der Kommunikationsmittel, deutsch in Communicatio Socialis 1 (1968), 343-346, 345 .

$8 \mathrm{~K}$. Hemmerle, Kommunikation in der Kirche - Kirche der Kommunikation, in: Communicatio Socialis 10 (1977), 253.

9 Ebd.

10 Auf sie verweist der Synodentext über die "Verantwortung des ganzen Gottesvolkes für die Sendung der Kirche $\propto$ in Nr. 3,2: Mitverantwortung wird ermöglicht und verwirklicht durch Kommunikation. Diese Kommunikation hat ihr Fundament im Verständnis der Kirche als eines Leibes mit vielen Gliedern, die durch Christus miteinander verbunden sind und um ihre Abhängigkeit voneinander wissen. Sie ist tätigwerdendes Offensein der Christen im Aufeinanderhören, im Miteinandersprechen, im Voneinanderlernen. *

11 Kienzler weist darauf hin, daß in der Dogmatischen Konstitution über die göttliche Offenbarung (Dei Verbum) das Wort 'communicare' benutzt wird, als davon die Rede ist, daß Gott sich selbst und seinen Heilsplan mit den Menschen kundgetan hat. *Damit wird der Offenbarungsvorgang zugleich auf die Höhe einer personalen Kommunikation ... gehoben , folgert Kienzler. A.a.O. $280 \mathrm{f}$.

12 Kienzlerbetont im Anschluß an Dei Verbum 10, daß Schrift und Tradition als die beiden Weisen der Offenbarung zwar zunächst der Weitergabe der rechten Lehre des Glaubens dienen, aber erst im Werk der Gemeinschaft, des Brotbrechens und des Gebetes (communicatione, fractione panis et orationibus) zu ihrer personalen Erfüllung kommen. A.a.O. 282.

13 Vgl. P. Watzlawik et alii., Menschliche Kommunikation. Formen, Störungen, Paradoxien. Bern, Stuttgart, Wien 4/1974. Der Autor macht dies an folgendem Beispiel deutlich: „Wenn Frau A auf Frau B's Halskette deutet und fragt: 'Sind das echte Perlen?', so ist der Inhalt ihrer Frage ein Ersuchen um Information über ein Objekt. Gleichzeitig aber definiert sie damit auch - und kann es nicht nicht tun - ihre Beziehung zu Frau B. Die Art, wie sie fragt (der Ton ihrer Stimme, ihr Gesichtsausdruck, der Kontext usw.) wird ent weder wohlwollende Freundlichkeit, Neid, Bewunderung oder irgend eine andere Einstellung zu Frau B ausdrücken. B kann ihrerseits nun diese Beziehungsdefinition akzeptieren, ablehnen oder eine andere Definition geben, aber sie kann unter keinen Umständen - nicht einmal durch Schweigen - nicht auf A's Kommunikation antworten. Für unsere Überlegungen wichtig ist die Tatsache, daß dieser Aspekt der Interaktion zwischen den beiden nichts mit der Echtheit der Perlen zu tun hat (oder überhaupt mit Perlen), sondern mit den gegenseitigen Definitionen ihrer Beziehung, mögen sie sich auch weiter über Perlen unterhalten. * (S. 54).

14 Ebd. 56.

15 Sehr ausführlich beschreibt Kienzler die Kirche als eine Kommunikationsgemeinschaft, als welche sie in LG aufscheint. Danach ist I. das Urbild der Kirche die göttliche Communio (nach LG 4: »So erscheint die ganze Kirche als 'das von der Einheit des Vaters und des Sohnes und des Heiligen Geistes her geeinte Volk' '). 2. Die Kommunikationsgemeinschaft der Kirche ist das 'Volk Gottes', womit die Kirche in die größeren Zusammenhänge der Heilsgeschichte und der messianischen Verheißungen hineingestellt wird. 3. Communio drückt auch das Verhältnis zwischen katholischer und anderen Kirchen aus, das als Kommunikationsgemeinschaft bezeichnet wird und 4 . ist die Verfassung der Kirche nach innen als «Communio hierarchica* charakterisiert, nach der die verschiedenen Ämter und Dienste ein »großes Gegenüber und Mitsammen selbständiger kommunikativer Personen« bilden. A.a.O. $283 \mathrm{ff}$.

16 F.J. Stendebach, Theologische Anthropologie des Jahwisten, Diss. Bonn 1970, 221 f., zit. nach F. Böckle, Fundamentalmoral, München 2/1978, 127.

17 P.M. Zulehner, Umkehr: Prinzip und Verwirklichung. Am Beispiel Beichte. Frankfurt/M. 1979, 79 f.

18 Vgl. ders., Helft den Menschen leben. Für ein neues Klima in der Pastoral. Freiburg 1978, 35.

19 Vgl. E. Feifel, Kommunikative Theologie. Rückfragen aus den «Erfahrungen mit dem Bußsakrament $*$ an die theologische Ausbildung, in: K. Baumgartner (Hrsg.), Erfahrungen mitdem Bußsakrament Bd. II, München 1979, 475-495, 485. Das macht beispielsweise auch die Ausübung der Ex-Kommunikation als Kirchenstrafe sinnenfällig. Sie ist die äußere Vollstreckung eines bereits innerlich vollzogenen Vorgangs: der Ausschlußaus 
derGemeinschaft. Durch schwere Schuld schließt sichder Täter selbst aus der Gemeinschaft sowohl mit Gott als auch mit der Gemeinde aus (vgl. Tit 3,10).

20 Communio et Progressio 8.

21 H. Küng, Christ sein. München, Zürich 3/1974, 466.

22 A.a.O. 279.

23 Alfons Deissler, Antworten des Alten Testaments, in: Wer ist das eigentlich, Gott?, hrsg. von H.J. Schultz, München 1969, 106.

24 J. Ratzinger, Einführung in das Christentum. München $3 / 1968,89$.

25 R. Schulte in Mysterium Salutis Bd. III 1, 78.

26 Communio et Progressio 11.

27 B. Häring, Theologie der Kommunikation und theologische Meinungsbildung, in: Kirche und Publizistik. Dreizehn Kommentare zur Pastoralinstruktion 'Communio et Progressio' mit dem deutschen Originaltext, hrsg. von F. J.Eilers u. a., Paderborn 1972, 38-44, 38.

28 B. Klaus, Einführung in die theologische Theorie kirchlicher Kommunikation, in: ders., Kommunikation 22.

29 Vgl. Ratzinger, Einführung, 191.

30 Nach Kienzler erfüllt sich Jesu Kommunikationswille in der Gabe des Heiligen Geistes: »Jesu Kommen ist der neue Anfang der Gemeinschaft des Menschen mit Gott und zugleich die Verheißung des immerwährenden Beistandes des Heiligen Geistes. Der Heilige Geist ist es, der die begonnene Gemeinschaft weiterträgt und lebendig hält. * A.a.O. 279.

$31 \mathrm{Vgl}$. Augustinus, De Trinitate XV 15, 16.

$32 \mathrm{Vgl}$. H. Mühlen, Soziale Geisterfahrung als Antwort auf eine einseitige Gotteslehre, in: Heitmann/Mühlen (Hrsg.), Erfahrung und Theologie des Heiligen Geistes, 253-272, 267.

33 Communio et Progressio 11. 


\section{SUMMARY}

The author tries to work out theological implications of the term $*$ communication « from God's way of acting and from the term »communio".

Communication establishes a relation and is thus image and adoption of God's communio to which it refers and which it anticipates in form of signs. Every successful act of communication is faith which consists in the vertical communication between God and man and the horizontal communication among the human beings. Its aim is human self-communication culminating in love and man's openness for God's message.

The writer also deals with the various steps of how God passes his word on to mankind, from the Old Testament to his final word that he has spoken though his Son. The ultimate unification of man is eventually brought about by the Holy Ghost who is the link that communio forms between the Father and the human beings who have been redeemed by his Son and thus been made his sons too.

\section{RÉSUMÉ}

Lauteur essaie de dégager les implications théologiques de la notion de communication à partir de l'oeuvre de Dieu et de la notion de »Communion «. La communicatin implique une relation et, ainsi, est une image et assimilation de la communion avec Dieu à laquelle elle renvoie et qu'elle anticipe comme un signe. Chaque communication réuissie est une croyance qui consiste en une communication verticale entre Dieu et les hommes et une communication horizontale des hommes entre eux. Son but est celui de la communication spirituelle culminant dans l'amour des hommes les uns pour les autres et aussi de leur ouverture à la communication spirituelle avec Dieu.

L'auteur prend en considération l c différentes étapes de la communication spirituelle de Dieu avec les hommes, depuis l'Ancien Testament jusqu'aux paroles finales de Dieu aux hommes qu'il a transmis aux hommes par l'intermédiaire de son fils.

L'Esprit Saint, qui est le lien, opère l'ultime conciliation avec les hommes: La communication réalisée entre le père et les hommes rachetés par le fils qui sonst, par ce moyen, fait ses enfants.

\section{RESUMEN}

El autor trata de poner de relieve, implicaciones teológicas del concepto de comunicación, a partir de la obra de Dios y de la idea de *communio". La comunicación produce una relación y es así una imagen y apropiamiento de la communio divina, a la cual remite y anticipa marcadamente. Cualquiera comunicación lograda es fe, que existe en la comunicación vertical entre el hombre y Dios, y en la comunicación horizontal de los hombres entre sí. Su finalidad es la propia participación entre los hombres mismos y que culmina en el amor, y su apertura a la propia participación de Dios.

El autor también trata el asunto de las diferentes etapas de la propia comunicación de Dios a los hombres, desde el AT hasta la definitiva palabra divina a los seres humanos, que habló a través de su Hijo. El último acuerdo de los hombres, lo consigue el Espiritu Santo, que es el lazo que establece la communio entre el Padre y los hombres redimidos por el Hijo, y que así de este modo también los hace hijos. 